\title{
Magnetic brain activity evoked and induced by visually presented words and nonverbal stimuli
}

\author{
CARSTEN EULITZ, ${ }^{\mathrm{a}, \mathrm{c}}$ HIND EULITZ, ${ }^{\mathrm{a}}$ BURKHARD MAESS, ${ }^{\mathrm{b}}$ RUDOLF COHEN, ${ }^{\mathrm{a}}$ \\ CHRISTO PANTEV, ${ }^{\mathrm{c}}$ AND THOMAS ELBERT ${ }^{\mathrm{a}}$
}

${ }^{a}$ Department of Psychology, University of Konstanz, Germany

${ }^{b}$ Max-Planck-Institute for Cognitive Neuroscience, Leipzig, Germany

${ }^{\mathrm{c}}$ Institute for Experimental Audiology, University of Münster, Germany

Konstanzer Online-Publikations-System (KOPS)

URL: http://www.ub.uni-konstanz.de/kops/volltexte/2006/2139/ URN: http://nbn-resolving.de/urn:nbn:de:bsz:352-opus-21398

\begin{abstract}
Evoked and induced magnetic brain activity measured over the left hemisphere were tested for their specificity to language-related processing. Induced activity refers to oscillatory alterations time locked but not phase locked to the stimulus. Words, false font stimuli, and two types of nonverbal patterns were presented visually while subjects performed a nonlinguistic visual feature detection task. The comparison of evoked and induced brain activity around $200 \mathrm{~ms}$ after stimulus onset revealed differential sensitivity to the stimuli. The M180 component of the evoked magnetic field was larger at the processing of words and false font stimuli compared with nonverbal stimuli. The induced magnetic brain activity in the $60-\mathrm{Hz}$ band at a compatible latency range was correlated with the familiarity of the visual Gestalt. Sensitivity to language-specific information processing can be concluded if a parameter differentiates the word condition from the nonlexical conditions. Such a difference was observed at sensors located over the frontal-temporal scalp regions for induced but not evoked magnetic brain activity. Thus, evoked and induced magnetic brain activity revealed a differential sensitivity to elements of cognitive processing during the given task.
\end{abstract}

Descriptors: MEG, Visual evoked magnetic field, Language, Lexical processing, Gammaband activity, Oscillatory brain activity

The sensitivity of event-related potentials (ERP), event-related fields (ERF), and electrocorticograms to various aspects of language processing has been reported in many studies (for review see $\mathrm{Ku}-$ tas, 1997; Kutas \& Van Petten, 1988). Early components before $300 \mathrm{~ms}$ have been shown to differentiate word processing from processing of visual information contained in objects, faces, or symbols (Allison, McCarthy, Nobre, Puce, \& Belger, 1994; Kuriki, Takeuchi, \& Hirata, 1998; Nobre, Allison, \& McCarthy, 1994; Schendan, Ganis, \& Kutas, 1998). Differences in the processing of words and nonwords were reported mainly after $200 \mathrm{~ms}$. The intracranially recorded N200 differentiated letter strings from faces and other objects but not words and nonwords (Nobre et al., 1994). During these intracranial recordings, a word-nonword difference was reported around $400 \mathrm{~ms}$. Similarly, the magnetoencephalographic (MEG) study by Salmelin, Service, Kiesilä, Uutela, and Salonen (1996) reported a word-nonword difference only in later portions of the ERF waveforms around $400 \mathrm{~ms}$. Furthermore, the

Research for this study was supported by the Deutsche Forschungsgemeinschaft and a grant from the Human-Frontier and Science program.

We thank the editor, S. Heim, and the unknown reviewers for helpful comments. We also thank L. Green for editing.

Address reprint requests to: Dr. Carsten Eulitz, Department of Psychology, PF 5560-D25, University of Konstanz, Universitätsstraße 10, D-78434 Konstanz, Germany. E-mail: Carsten.Eulitz@uni-konstanz.de. results of Pulvermüller, Lutzenberger, and Birbaumer (1995) and Schendan et al. (1998) are compatible with the idea that words and nonwords are not differentiated until after $200 \mathrm{~ms}$. Given these results, the electrophysiological brain activity until after $200 \mathrm{~ms}$ probably reflects prelexical processing of words or letter strings. This conclusion needs, however, additional research because lexical processing has also been demonstrated for that latency interval. For example, Pulvermüller et al. (1995) reported an ERP component around $160 \mathrm{~ms}$ that differed between the processing of function and content words.

Event-related responses are extracted by averaging the electroencephalogram (EEG) or MEG across repeated trials. In addition to the analyses of this evoked brain activity, frequency-domain analysis of EEG and MEG data has been introduced as a further tool for quantifying functional changes in neural activity (Makeig, 1993). This approach estimates the induced brain activity based on time- but not phase-locked event-related activity and thus reveals aspects of brain activity that are not visible when using timedomain analysis. A number of studies (for review see Pulvermüller, Birbaumer, Lutzenberger, \& Mohr, 1997; Tallon-Baudry \& Bertrand, 1999) have demonstrated the suitability of induced brain activity parameters from the high-frequency range (above $20 \mathrm{~Hz}$ ) for the description of specific cognitive processes, in particular for elements of language processing. Pulvermüller and co-workers (Pulvermüller, 1996; Pulvermüller et al., 1997) proposed that the 
induced brain activity in the high-frequency range may reflect the activation of neuronal assemblies specific for certain elements of cognitive processing with defined cortical topographies and temporal activity patterns. The variety of the reported frequency ranges and latencies in only a small number of studies, together with the range of experimental designs used, require additional research to further illuminate the role of high-frequency responses during language processing. For this purpose, two major issues will be studied. First, is there a high-frequency response specific to aspects of word processing? If so, what are the frequency bands and time ranges of relevance? The second issue is whether parameters of the evoked and the induced brain activity reflect the same or different aspects of information processing.

Using analyses of the induced neuromagnetic activity, we have previously reported enhanced normalized spectral power around $200 \mathrm{~ms}$ in the $60-65-\mathrm{Hz}$ band over the left hemisphere when subjects processed language-related material and over the right hemisphere for a nonlanguage condition (Eulitz et al., 1996). The result was independent of modality (auditory or visual) of stimulus presentation. It was concluded that this enhancement of the normalized spectral power reflected oscillatory patterns specific to the processing of words. To further explore the functional role of this oscillatory brain activity, in addition to the stimuli used in the previous investigation, namely, word and false font stimuli (four rotated, mirrored, or distorted letters), two further nonverbal stimulus classes were used in this study: shapes (complex pattern with a row of four or five clusters of connected pixels) and dots stimuli (randomly arranged pixels on an area identical to the other classes of stimuli).

As in the previous study, we used the MEG for these measurements specifically because it is a reference-free signal and, in certain conditions, may have a signal-to-noise ratio superior to that from the EEG (Eulitz, Eulitz, \& Elbert, 1997). As previously, subjects had to detect targets that appeared occasionally in a series of standard (nontarget) stimuli. The task was to detect a common nonlinguistic feature in all four classes of stimuli. According to the "word superiority effect" meaningful words are processed differently from nonwords and nonsense letter strings, even when processing is not required by the task (Reicher, 1969; Wheeler, 1970). Posner, Sandson, Dhawan, and Shulman (1989) showed that words automatically activate systems for visual word form recognition. Furthermore, positron emission tomography (PET) studies have shown the passive processing of visually presented words to be sufficient to activate additional brain structures as compared with the processing of nonverbal stimuli (Petersen, Fox, Snyder, \& Raichle, 1990; Price, Wise, \& Frackowiak, 1996). Among other regions, differential activation was demonstrated in the left prefrontal region for words versus nonwords.

In sum, it was expected that especially for passive processing conditions, the processing of lexical stimuli should be focused in distinct and specialized subsystems of the brain. If this languagerelated information processing is correlated with the activation of neuronal assemblies specific for that processing, we should be able to describe it by means of a specific topographic and temporal activity pattern of the induced brain activity in the high-frequency range for the nontarget stimuli. The main hypothesis regarding the processing of visual information in the four different classes of complex stimuli is the lexical hypothesis, which assumes that lexical information is processed differently from nonlexical information. Another possibility is that differences in brain activation will reflect the cumulative experience in processing of visual information (see also Schendan et al., 1998). The more connected the pixels and the more related they are to shapes and forms of familiar Gestalts, the larger will be the change of brain activational parameters related to Gestalt perception. Such a Gestalt hypothesis assumes that the brain activation should be smallest for the dots condition, larger for the shapes condition, then the false font condition, and largest for the word condition.

\section{Materials and Methods}

\section{Subjects}

Fifteen right-handed (Oldfield, 1971) subjects (4 female, 11 male) aged between 20 and 32 years (median: 26.5 years) participated in the study. None of the first-degree family members (parents, siblings) were reported to be left handed. Two subjects were excluded from further analysis due to a very low signal power of MEG responses.

\section{Stimuli and Procedure}

Four different classes of complex visual stimuli, equated in luminance, mean number of pixels, and area covered by the patterns, were used. The classes of visual stimuli differed in degrees of complexity and concepts. The following stimulus classes were used: (a) word stimuli-content words with two syllables (four characters); (b) false font stimuli-pattern of four rotated, mirrored, or distorted letters, which did not allow for reconstruction into real German words; (c) shapes stimuli_complex pattern with a row of four or five clusters of connected pixels; and (d) dots stimuli-randomly arranged pixels on an area identical to the other classes of stimuli. For each stimulus class 42 variants of stimuli were created. Examples are demonstrated in Figure 1.

From these stimuli, a subset of 21 different target stimuli for each stimulus class (i.e., 84 variants for the whole set of nontarget stimuli) was derived by removing a small section from the stimulus (see right column of Figure 1). The deletion could appear on variable positions within the stimulus and the average size of the removed part was different for the stimulus classes. As also seen in Figure 1, the deletion was smaller for the words than for the other stimulus classes. This procedure was chosen to equate error rates

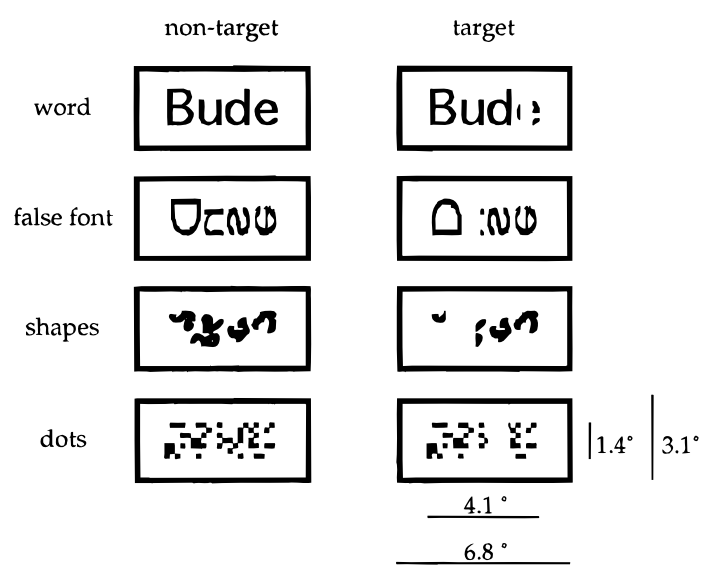

Figure 1. Examples of visual word, false font, shapes, and dots stimuli out of a set of 42 different variants per stimulus class are illustrated in the left column. Target stimuli are shown in the right column. The latter were derived from the nontarget stimuli by cutting out a small area of one of the characters. In the bottom part of the figure, the respective visual angles are indicated. 
for the different classes. ${ }^{1}$ The subject's task was to detect the targets and to respond by pressing a button as accurately and quickly as possible. The probability of a target appearing was $15 \%$ for all stimulus conditions.

The experiment consisted of six blocks of trials. For each block, two of the four stimulus classes were presented in random order. Each stimulus class was combined with each of the others across blocks. The order of recording blocks was balanced across subjects and each pair of stimulus conditions occurred equally often at each position. Each stimulus class had the same probability of occurrence in two subsequent recording blocks. For each subject, a total of 252 stimuli per experimental condition were presented. This corresponds to six repetitions of each nontarget stimulus across three of the six experimental blocks during the measurements.

Visual stimuli were presented for $400 \mathrm{~ms}$ on a screen using nonmagnetic fiber optic transmission. The interstimulus interval varied randomly from 2.0 to $2.4 \mathrm{~s}$. The screen was positioned $60-70 \mathrm{~cm}$ in front of the subject's eyes. During the measurement, a frame (visual angles: $3.1^{\circ}$ vertical, $6.8^{\circ}$ horizontal) was displayed continuously on the screen, and subjects were asked to focus on the center of the frame. Stimuli were presented in the center, with a maximum of $1.4^{\circ}$ vertical, and $4.1^{\circ}$ horizontal extremum.

Before the experiment, it was assured that all participants were able to identify all words and target stimuli with an accuracy of $100 \%$. During the preparation for the measurement and during the instructional session, the stimuli were presented repeatedly to the subjects to familiarize them with the task and to minimize a possible impact of repetition effects later during the experimental session. Using this procedure, the influence of memory effects cannot be ruled out, as differential ERP repetition effects for words and nonlanguage patterns are possible (Van Petten \& Senkfor, 1996). However, in addition to the familiarization with the stimuli, the experimental session contained six repetitions, the task for the subjects was target detection, the subjects were not instructed to memorize any stimuli, and there was no relevance of the stimulusclass-related visual information in the nontarget stimuli for any aspects of the experimental session.

\section{MEG Measurements}

Neuromagnetic data were recorded using a 37-channel neuromagnetometer (Magnes, Biomagnetic Technologies, Inc.; consisting of first-order axial gradiometers with 5-cm baseline; pick-up coils are arranged in an array of concentric circles with a diameter of $14.4 \mathrm{~cm}$ ). Measurements were carried out in a magnetically shielded room. The sensor array was centered over the left supratemporal cortex about $1.5 \mathrm{~cm}$ superior to the T3 electrode position of the 10-20 system. The head position relative to sensor pickup coils was measured by a sensor position indicator. Horizontal and vertical electrooculogram (EOG) and the button press was also recorded. ${ }^{2}$ Subjects were lying on their side with the head fixated by a vacuum cushion. They were instructed to avoid eye blinks and head movements, and to carry out the monitoring task carefully. Con-

\footnotetext{
${ }^{1}$ Tests with equal sized removed sections in target words and "false font" target stimuli prior to the experiment revealed a systematically lower error rate in the "word" (mean error rate: $3 \%$ ) as compared with the "false font" condition (16\%). Consequently, the average size of the removed parts of the target stimuli was enlarged in all nonlanguage target stimuli to equalize the error rate across stimulus classes.

${ }^{2}$ In addition, EEG was recorded from F3, F4, T3, T4, P3, P4, M1, and M2 referred to $\mathrm{Cz}$. Results focusing on EEG and MEG comparisons are reported in Eulitz, Eulitz, and Elbert (1997).
}

tinuous data were recorded in 7-8-min blocks at a sampling rate of $297.6 \mathrm{~Hz}$ within a bandpass width of $0.03-100 \mathrm{~Hz}$.

\section{Data Analysis}

Averaged waveforms for nontargets were calculated for each stimulus class across epochs of 1,500 ms, including a 300-ms prestimulus baseline. Artifact-contaminated epochs with a signal deviation of more than $2.5 \mathrm{pT}$ in the MEG, or $120 \mu \mathrm{V}$ in the EOG, and epochs with an erroneous button press were excluded. The averaged waveforms were digitally filtered using a lowpass filter of $20 \mathrm{~Hz}(12 \mathrm{~dB} /$ oct $)$.

Peak latencies and peak amplitudes were defined for several ERF components. They were the maximum signal deviation from baseline in the following time intervals poststimulus onset: (1) M100: 90-135 ms; (2) M180: 160-220 ms. For better comparability between time- and frequency-domain-based results, the mean M180 amplitude for the latency range from 160 to $220 \mathrm{~ms}$ was calculated and used for statistical analysis instead of just the peak amplitude. Although literal comparability cannot be achieved, this procedure results in an amplitude measure representing integrated time information similar to the parameter extracted for the spectral intensities. For comparisons, the signal power was determined as the root-mean-squared (rms) value across all MEG channels over the left hemisphere.

For frequency-domain analyses, a method similar to eventrelated perturbation analyses (Makeig, 1993) was applied using the avg_q software (Feige, 1999). In artifact-free epochs of MEG raw data (same rejection criteria as reported above), power spectral estimates were derived from Fourier transforms on pairs (overlapping by one half) of 214-ms Welch-windowed segments (one half of the segment contained data, whereas for the other half zero padding was used to enhance the frequency resolution; the lowest frequency bands were therefore insufficient for analysis). Under these constraints, a frequency resolution of $4.6 \mathrm{~Hz}$ was obtained. Power estimates were selectively averaged for each segment around stimulus onset and stimulus class. Nine time segments were situated equidistantly within a 600-ms interval before stimulus onset (baseline), and 24 segments after stimulus onset within the total interval of 1,343 ms. Only spectral power changes in frequency bands above $7 \mathrm{~Hz}$ were considered. The mean power spectra were transformed with respect to baseline-related changes. Normalized mean power spectra were calculated by dividing each single mean spectral power within one time-frequency bin by the mean spectral power across all corresponding baseline segments.

To further reduce the data and to obtain estimates that were not affected by small alterations in the location or orientation of the generating brain regions, and that showed little dependency on individual variations of brain anatomy, the normalized spectral power was collapsed across the 37 recording channels. This method led to a smoothing and reduction of effects when significant changes in spectral power were only local. However, the mean differences between experimental conditions were small and using robust parameters and conservative statistical testing was preferred. For a more detailed topographical analysis of effects found with the conservatively reduced data, three subaverages each consisting of 12 magnetic channels were calculated. The channels were chosen over the frontal-temporal, the central-parietal and the temporaloccipital scalp regions (see also Figure 6). Across subjects, the sensor groups were aligned with the middle channel of the sensor array (positioned $1.5 \mathrm{~cm}$ superior to T3), and with the most superior channel of the sensor array (which was close to $\mathrm{Cz}$ ). For the comparison of parameters from time- and frequency-domain analy- 
ses, the same subaverages were calculated using the absolute values of the mean M180 amplitude.

Statistical analyses were carried out using univariate analyses of variance (ANOVA). Where appropriate, Greenhouse-Geisser adjustments were carried out. The peak latencies and rms amplitudes of the ERF components, and the normalized spectral power were used as dependent variables. Repeated measures one-way ANOVA were used to compare the four stimulus classes (false font vs. shapes vs. dots vs. word). Regional normalized spectral powers were statistically tested with a 4 Stimulus Classes (false font vs. shapes vs. dots vs. word) $\times 3$ Sensor Groups (frontal-temporal vs. central-parietal vs. temporal-occipital) design (repeated measures). For a comparison of parameters from the time- and the frequencydomain analyses, each parameter was $z$-transformed across all stimulus classes, sensor groups, and subjects. The $z$-transformed parameters were then used for a repeated-measures three-way ANOVA for a 2 Methods (time- vs. frequency-domain analysis) $\times 4$ Stimulus Classes $\times 3$ Sensor Groups design.

Planned comparisons using contrasts were calculated for post hoc analyses. Three different hypotheses about factors influencing the expression of dependent variables were tested: (a) the lexical hypothesis, that is, word differs from all three other stimulus classes (contrasts: word [3], false font $[-1]$, shapes $[-1]$, dots $[-1])$; (b) the verbal hypothesis, that is, subjects may be able to verbalize false font stimuli or parts of them after some simple mental transformation, leading to a verbalization in both the word and the false font condition but not in the shapes and dots condition (contrasts: word [1], false font [1], shapes [-1], dots [-1]); and (c) the Gestalt hypothesis, that is, the more connected the pixels to shapes and forms and the more related the shapes and forms to known concepts or Gestalts, the larger the change of a parameter indicating activational processes involving binding of perceptual features to Gestalts will be. In terms of contrasts, the Gestalt hypothesis can be tested using a linear model (contrasts: word [3], false font [1], shapes $[-1]$, dots $[-3]$ ). As the contrasts for the three hypotheses are not orthogonal, for planned comparisons the significance level $(\alpha=0.05)$ was adjusted to $\alpha^{\prime}=0.0125$ by means of the Bonferroni procedure.

\section{Results}

\section{Evoked Activity}

Figure 2 presents the averaged MEG waveforms of one subject for the different experimental conditions. The grand averages (across all subjects) of MEG signal power (rms waveforms) are illustrated in Figure 3. After the initial M100 and M180, which may correspond to the electric P100 and N180, a slow component lasting up to $600 \mathrm{~ms}$ after stimulus onset appears under all experimental conditions. This component probably results from the sustained activity during stimulation and from more stimulus-specific information processing. The mean latencies of the components did not differentiate experimental conditions. However, mean rms amplitude began to differ between experimental conditions about $150 \mathrm{~ms}$ after stimulus onset. The M180 component was larger for the false font and the word conditions than for the shapes and the dots conditions.

The ANOVA comparing the four stimulus classes (false font vs. shapes vs. dots vs. word) revealed significant amplitude-differences with no latency-differences for the M180. The mean M180 rms peak amplitude was larger for the false font (125.9 fT) and word (125.3 fT) conditions than for the shapes (98.3 fT) and dots $(104.7 \mathrm{fT})$ conditions, $F(3,36)=7.88, \epsilon=0.636, p<.003$. The verbal hypothesis, $F(1,12)=14.23, p<.003$, explained the differences between conditions.

\section{Induced Activity}

An overview of stimulus induced changes of normalized spectral power in the whole time-frequency area averaged across all subjects is shown in Figure 4. In three time-frequency regions, the spectral power was enhanced relative to baseline and one time/ frequency region showed a reduction of spectral power under all experimental conditions. Enhanced spectral power around $10 \mathrm{~Hz}$ was obvious from 50 to $200 \mathrm{~ms}$, around $60 \mathrm{~Hz}$ from 100 to $400 \mathrm{~ms}$, and around $10-40 \mathrm{~Hz}$ from 700 to $1,100 \mathrm{~ms}$. The enhancement around $60 \mathrm{~Hz}$ from 100 to $400 \mathrm{~ms}$ seems to be most pronounced in the word condition and is absent in the dots condition (see also Figure 5 for an extended view of the normalized spectral power changes for the $60-\mathrm{Hz}$ band as a function of time). The late enhancement of spectral power from 700 to $1,100 \mathrm{~ms}$ was most pronounced in the shapes and dots condition. Only in the dots condition did this late enhancement tend to be enlarged in lower frequency bands. A reduction of normalized spectral power is seen around $200-600 \mathrm{~ms}$ in the $10-40-\mathrm{Hz}$ range. This reduction of spectral power was on average somewhat stronger in the shapes condition as compared with the other experimental conditions.

The essential change of spectral power for the present report ${ }^{3}$ was the enhancement around $60 \mathrm{~Hz}$ from 100 to $400 \mathrm{~ms}$. In the word condition, the $60-\mathrm{Hz}$ enhancement was largest at the timefrequency window centered at $183 \mathrm{~ms}$, where it markedly exceeded the variability during the baseline interval (Figure 5). No changes of spectral power were evident for the dots condition. The false font and the shapes conditions also showed enhancements of spectral power in that time-frequency bin but those enhancements were smaller. ANOVA revealed a significant main effect of stimulus classes for the spectral power, $F(3,36)=3.13, \epsilon=0.838, p<$ .05 . Post hoc planned comparisons suggested that the Gestalt hypothesis explains the spectral power differences in the $60-\mathrm{Hz}$ band, $F(1,12)=10.77, p<.007$.

For a more detailed topographical analysis of the effects within the $60-\mathrm{Hz}$ band, three subaverages for 12 magnetic channels each, grouped over the frontal-temporal, the central-parietal, and the temporal-occipital regions were calculated. As can be derived from Figure 6, changes of spectral power over central-parietal and temporal-occipital sensor groups were similar to the overall changes displayed in Figure 5. Over the frontal-temporal sensor group, enhanced spectral power was obtained only in the word condition.

Regional normalized spectral power showed statistically significant main effects for sensor groups, $F(2,24)=3.91, \epsilon=0.958$, $p<.04$, as well as stimulus classes, $F(3,36)=3.43, \epsilon=0.823$, $p<.05$. The sensor groups effect was primarily explained by the reduced spectral power over frontal-temporal areas in the false font and shapes condition. Planned comparisons for explanation of the stimulus classes effect showed the same result as the analyses across all channels. The Gestalt hypothesis explains the spectral

\footnotetext{
${ }^{3}$ Spectral power changes for other time frequency windows also showed statistically significant differences between stimulus classes. The reduction of normalized spectral power was largest in the $19 \mathrm{~Hz}$ frequency band at the time window centered at $291 \mathrm{~ms}, F(3,36)=3.50, \epsilon=0.773, p<.05$. The late enhancement of spectral power from 700 to $1,100 \mathrm{~ms}$ in the $10-40-\mathrm{Hz}$ frequency band was seen in all experimental conditions and tended to indicate differences between stimulus classes mainly in the frequency range below $25 \mathrm{~Hz}, F(3,36)=3.58, \epsilon=0.665, p<.05$, around $13.9 \mathrm{~Hz}$ and $830 \mathrm{~ms}$. However, these changes were not essential for the questions under investigation and will not be discussed in detail.
} 


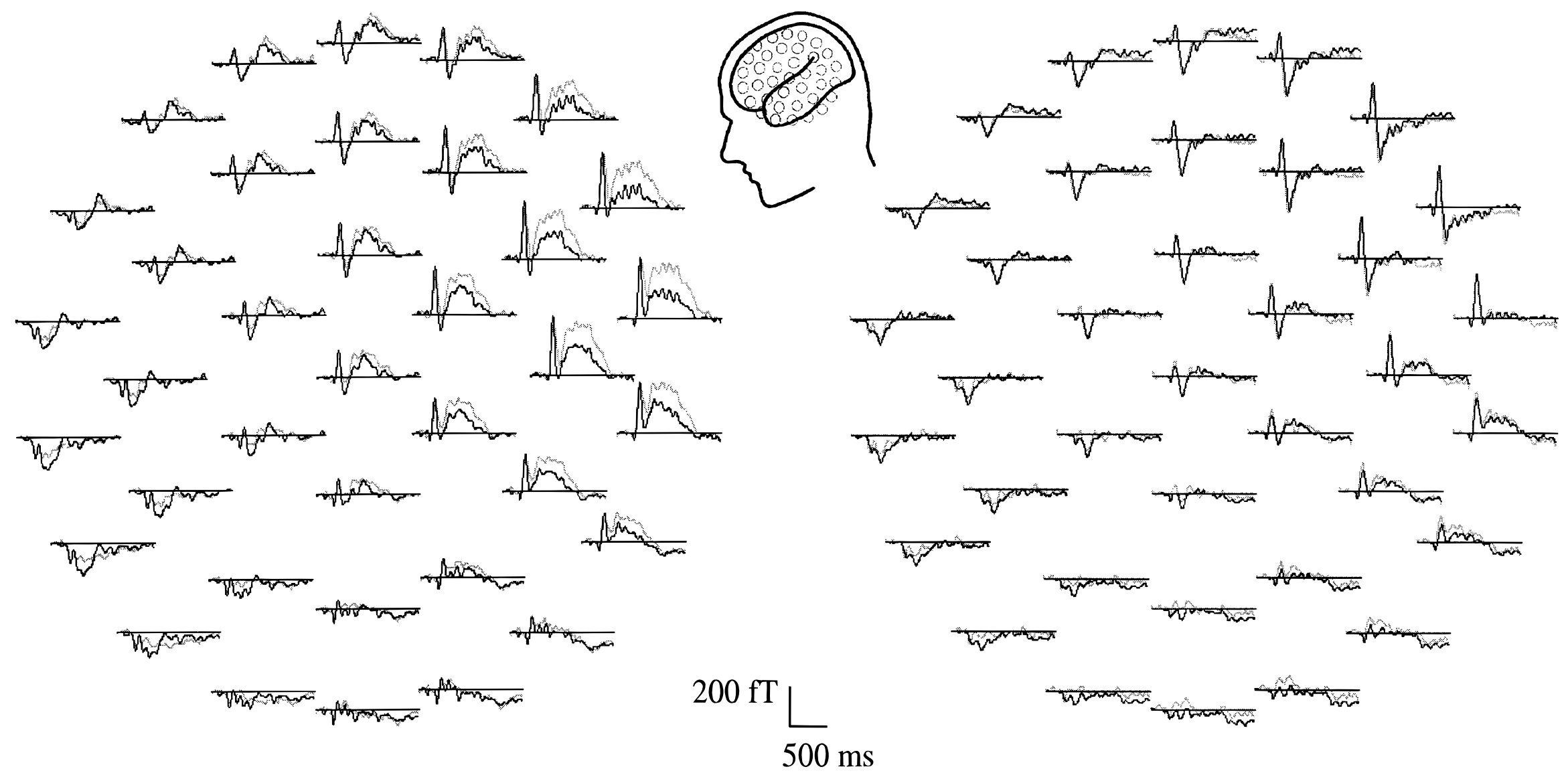

Figure 2. Typical magnetoencephalographic waveforms evoked by the different classes of stimuli are shown for subject S11. Data were collected simultaneously for all 37 channels over the left hemisphere. The approximate locations of the pick-up coils relative to the subject's head are shown schematically. The left panel shows the word (solid line) and the false font (dotted line) condition. The right panel presents the shapes (solid line) and the dots (dotted line) condition. 


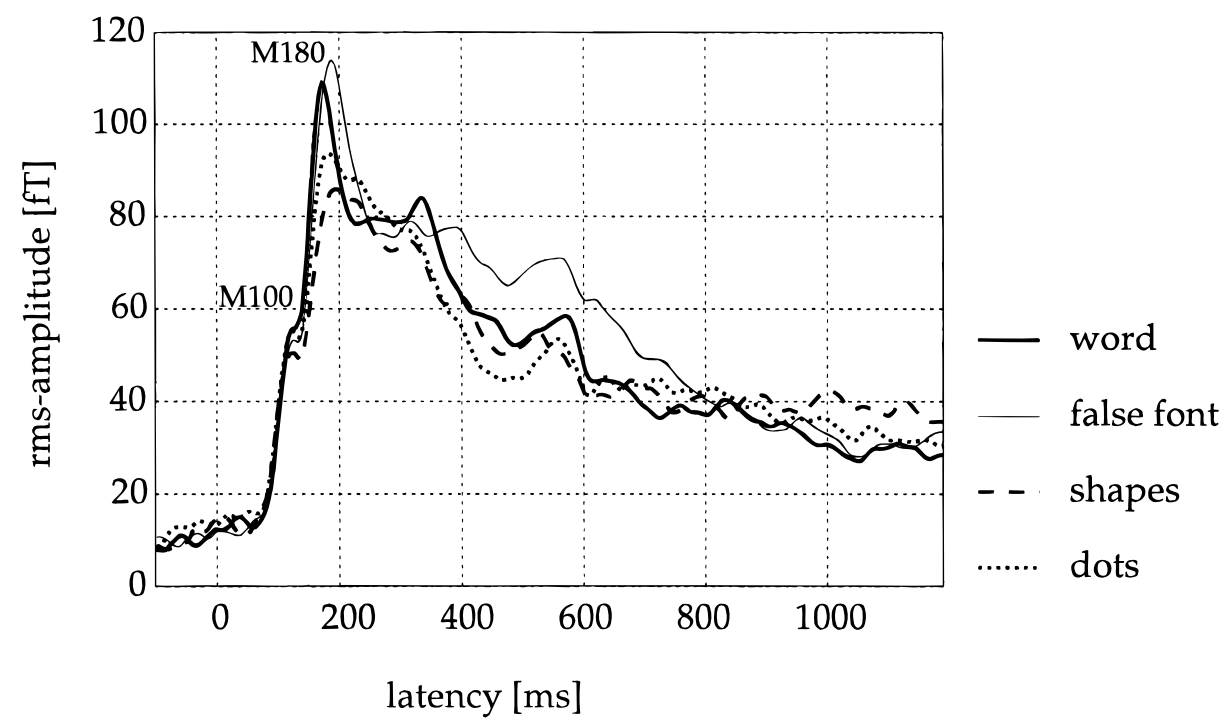

Figure 3. Grand-average root mean squared (rms) waveforms recorded from the left hemisphere are shown separately for the different experimental conditions. The thick solid line codes the word condition, the thin solid line the false font condition, the dashed line the shapes condition, and the dotted line the dots condition. Only $100 \mathrm{~ms}$ of the $300-\mathrm{ms}$ baseline are plotted in this figure.

power differences in the $60-\mathrm{Hz}$ band. Testing the hypotheses separately for a sensor group of interest (for arguments see Discussion) indicated that over the frontal-temporal sensor group, the lexical hypothesis, $F(1,12)=18.14, p<.002$, and the Gestalt hypothesis, $F(1,12)=11.39, p<.002$, explain spectral power differences in the $60-\mathrm{Hz}$ band. The corresponding mean spectral power differences between stimulus classes (see Figure 6) support the lexical hypothesis.

\section{Comparison Between Evoked and Induced Activity}

The effect in the $60-\mathrm{Hz}$ band that may reflect aspects of language processing occurs in a similar time interval as the M180 component of the evoked activity. To answer the question as to whether or not both parameters reflect the same neurophysiological processes, the expression of both parameters for the different stimulus classes and sensor groups was compared. Figure 6 shows the $z$-transformed amplitude values of both parameters for all stimulus word
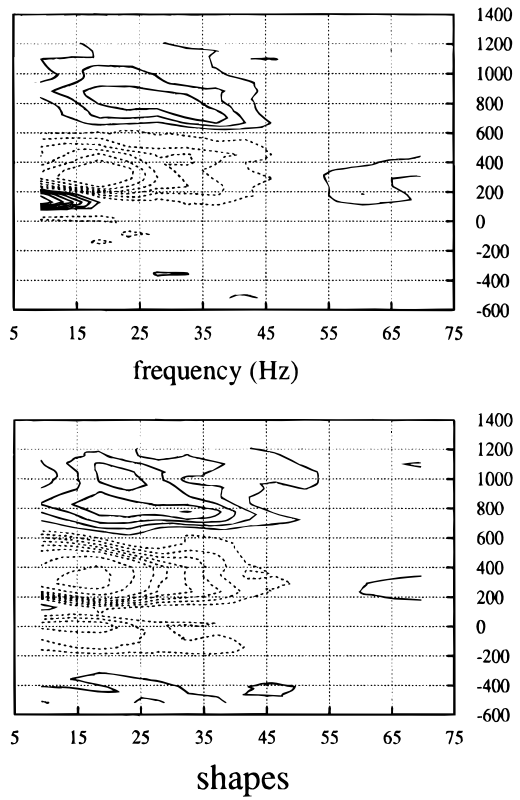

false font
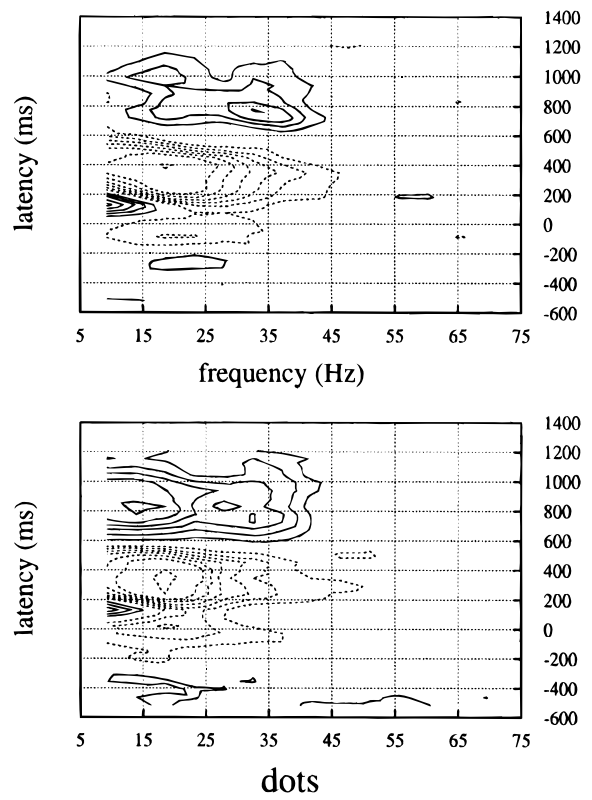

Figure 4. Normalized spectral power for all time/frequency bins averaged across all channels and then across all subjects is presented for all experimental conditions. The upper part of the figure displays the results from the verbal conditions, and the lower part those from the nonverbal conditions. In each picture, the $x$-axis represents the frequency, the $y$-axis the latency, and the level of normalized spectral power is indicated by isocontour lines. Solid lines are used for time/frequency areas of enhanced normalized spectral power by more than $1.5 \%$; dotted lines indicate a reduction of normalized spectral power by more than $1.5 \%$. The contour step is $\pm 1.5 \%$ up to $\pm 9 \%$ and then $\pm 3 \%$. 


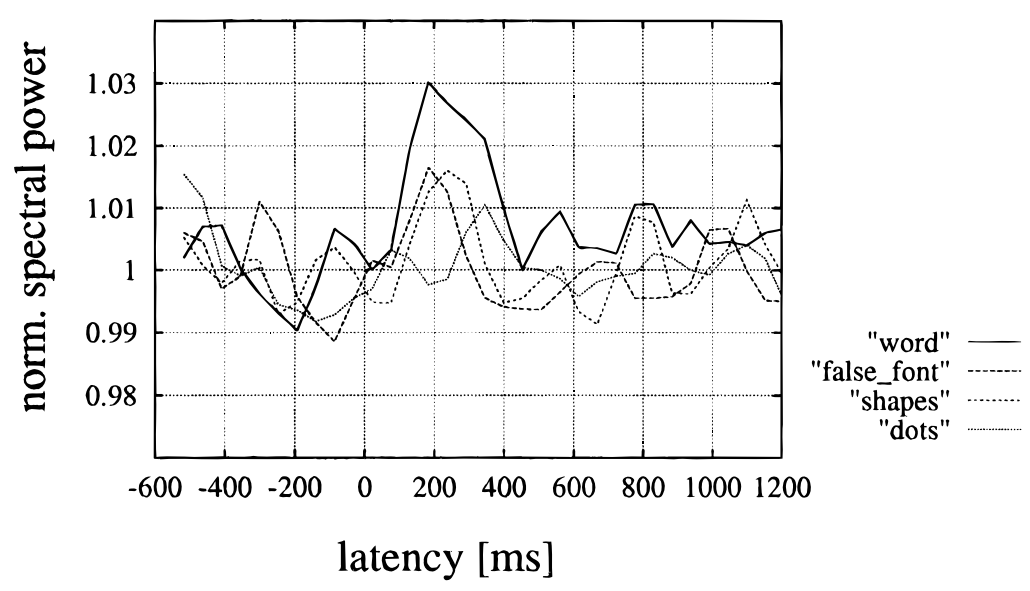

Figure 5. Changes of the normalized spectral power averaged across all channels and then across all subjects as a function of time for the frequency band around $60 \mathrm{~Hz}$ for all experimental conditions. The solid line codes the word condition, the long-dashed line the false font condition, the short-dashed line the shapes condition, and the dotted line the dots condition. A normalized spectral power of 1 corresponds to the mean spectral power across nine prestimulus time windows (separately calculated for each frequency band). A normalized spectral power of 1.05 indicates their enhancement by $5 \%$ above baseline level.

classes separately for three sensor groups. The mean M180 amplitude was larger for all stimulus classes over temporal-occipital scalp regions as compared with the other regions, especially for the false font condition. In contrast, this topography is not seen for the spectral power changes in the $60-\mathrm{Hz}$ band. Regional differences in the spectral power were evident for all stimulus classes except for the word condition. For the later condition, the enhancement of spectral power seemed to be widespread across the whole left hemisphere. The most characteristic feature for the comparison between experimental conditions emerged over the frontal-temporal region, where the spectral power was enhanced as compared with the baseline level only for the word condition.

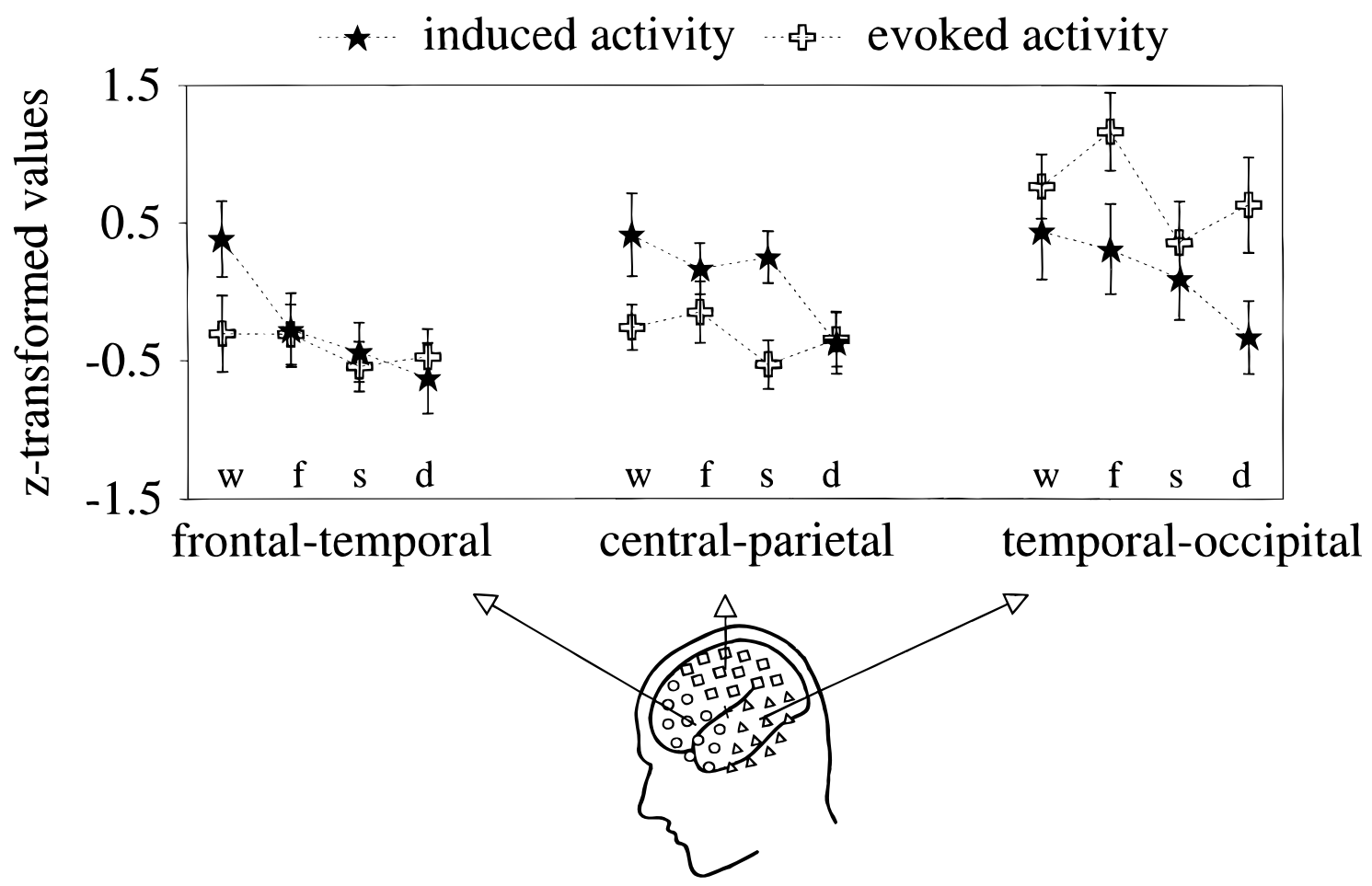

Figure 6. Mean $z$-transformed values of the normalized spectral power for the $60-\mathrm{Hz}$ effect (induced activity indicated by stars) and the mean M180 amplitude (evoked activity indicated by crosses) are shown. The three sensor groups of interest are presented separately in the columns. Within each column, the values for the stimulus classes are demonstrated in the following order: word (w), false font (f), shapes (s), and dots (d) condition. Corresponding parameters from the evoked and the induced brain activity are connected by a dotted line. Error bars indicate between-subject variability (standard errors). The schematic head in the bottom part of the figure shows the projection of the 37 channels of the used magnetoencephalograph (MEG) on the left side of a head. Different symbols indicate the channels used for subaverages of the parameters for more detailed topographical analyses. Circles represent the frontal-temporal, rectangles the central-parietal, and triangles the temporal-occipital sensor groups. Note the different rankings of the induced and the evoked activity for the sensor groups, and the different rankings for induced activity over the frontal-temporal sensor groups across the stimulus classes. 
Statistical analysis using stimulus class, sensor groups, and method as repeated-measures factors revealed in addition to significant main effects of the stimulus class, $F(3,36)=3.97, \epsilon=$ $0.834, p<.02$, and sensor group, $F(2,24)=15.03, \epsilon=0.833, p<$ .001 , also significant interactions of sensor groups and method, $F(2,24)=9.46, \epsilon=0.983, p<.001$, as well as stimulus class and method, $F(3,36)=3.21, \epsilon=0.854, p<.05$. The interactions confirmed that the parameters of the induced and the evoked brain activity differ in topographic distribution and were differently expressed for the investigated stimulus classes.

\section{Discussion}

To further illuminate the role of high-frequency oscillatory brain responses during language processing, we studied induced neuromagnetic activity in the high-frequency range during the processing of visually presented words and nonverbal stimuli and compared the differences between conditions with the differences observed in the parameters of the evoked neuromagnetic brain activity. The analyses were focused on the brain activity around $200 \mathrm{~ms}$. The interactions of both parameters with the sensor groups and with the stimulus class demonstrate that induced and evoked brain activity have a different topography and a different dependency of the signal power on the investigated stimulus classes. We conclude that the two parameters reflect different neurophysiological processes. This point is also illustrated by the observation that for both parameters different post hoc hypotheses explained the differences across conditions. The verbal hypothesis explained the differences across conditions in the evoked brain activity, whereas the Gestalt hypothesis explained the differences of the induced brain activity.

The point that both parameters reflect different neurophysiological processes is important for the following reason. Highfrequency induced brain activity has been proposed as an index of specific cognitive processes, and should be mediated by the activation of distributed neuronal assemblies specific for certain elements of cognitive processing (Pulvermüller, 1996; Pulvermüller et al., 1997). The Sensor Group $\times$ Method and the Stimulus Class $\times$ Method interactions in the present results indeed support the idea that the area-specific spectral power changes in the $60-\mathrm{Hz}$ band reflect different aspects of information processing as the evoked neuromagnetic activity. One reason for the differences might be that cognitive processes are time locked but not phase locked to the stimulus onset and will be systematically detected only with the frequency-domain analyses. Support for the assumption that aspects of language may generate a considerable jitter in the timing of information processing from word to word was demonstrated for the influence of word frequency during reading (King \& Kutas, 1998). In studies of cognitive processes in which a considerable jitter in the timing of information processing can be expected, analyses of the induced brain activity may be a valuable tool to reveal aspects of brain activity that are less visible in the evoked brain activity.

The evoked neuromagnetic activity reflected systematic differences in the processing of the stimulus classes around $200 \mathrm{~ms}$ after stimulus onset and thus confirmed previous results, which demonstrated differential processing of words and visual information in objects, faces, or symbols (Allison et al., 1994; Kuriki et al., 1998; Nobre et al., 1994). As the M180 amplitude did not differentiate the word condition from a variety of nonlexical conditions, the differences did not support the view that the information processing reflected in the M180 is language specific. It should be considered that M180 amplitude differences may also be influenced by differential repetition effects for words and nonverbal stimuli, as noted previously for later components in electrical recordings (Van Petten \& Senkfor, 1996). This could have affected the present results, even though there was a large number of repetitions during the instruction of participants and the experimental session.

The induced brain activity in the $60-\mathrm{Hz}$ band reflected systematic differences in the processing of the stimulus classes. The differences between the word and the false font conditions confirmed the earlier results of Eulitz et al. (1996), who reported a larger spectral power during automatic word processing. However, the overall differences across all stimulus classes under investigation when using measures from a sensor array that nearly covers the whole left hemisphere showed a dependency of event-related oscillations in the $60-\mathrm{Hz}$ band with the familiarity of the visual Gestalt and did not support the lexical hypothesis. This finding demonstrates that the presently introduced additional sets of control stimuli allow for a more concise interpretation of the $60-\mathrm{Hz}$ effect and the functional significance of parameters. Based on the overall changes of the induced activity, we may suggest that the parameter reflects information processing on the prelexical level. The activity seems to be "sensitive to the statistical regularities within well-learned, visual image categories" (Schendan et al., 1998, p. 249). Although this notion originates from examining the functional role of an ERP component, that is, from electric and not magnetic and from evoked and not induced brain activity, the present results support the generalization of this idea.

Brain imaging studies support the specific role of the left frontal operculum for word reading (Fiez \& Petersen, 1998). PET studies with tasks closely related to the experimental manipulations in the present study were reported by Petersen et al. (1990) and Price et al. (1996). In these studies, differential PET activational pattern for visual words and wordlike stimuli in a passive viewing situation and during the implicit processing ${ }^{4}$ of the visual information were observed. Compared with the nonlanguage conditions, an increased metabolic activation was reported in left inferior prefrontal cortex during the word condition. An activity in this brain area would mainly be measured by the frontal-temporal sensor group. Based on this evidence, a regional post hoc comparison seems straightforward and indeed showed that both the lexical hypothesis and the Gestalt hypothesis explained the differences across conditions. Mean spectral power differences between stimulus classes (see Figure 6) support the lexical hypothesis. It should be noted, however, that the Sensor Group $\times$ Stimulus Class interaction did not reach significance, indicating a high variability of regional pattern across subjects. Nevertheless, we may tentatively conclude that over the left frontal-temporal scalp region the high-frequency-induced magnetic brain activity in the $60-\mathrm{Hz}$ band in the 200-ms time segment reflects the activation of neuronal subsystems specialized for automatic word processing, which may be in part spatially distinct from those involved in the processing of the other stimulus classes. However, these statements are driven by assumptions derived from imaging studies. It is well known that electrophysiological and imaging methods reflect different aspects of brain activity and using blood flow to constrain the regions of neuromagnetic activity has to be considered with caution (Elbert, 1998; Eulitz et al., 1994; Stippich et al., 1998). Furthermore, a more precise location of the generators of the induced neuromag-

\footnotetext{
${ }^{4}$ Subjects performed a nonlinguistic feature detection task and were not instructed to process words and pseudowords. Price, Wise, and Frackowiak (1996) called the word and pseudoword processing implicit.
} 
netic activity is certainly highly desirable but was not attempted in the present study for the following reasons. The partial coverage available with the 37-channel system is small. At the same time, the scalp distribution of the magnetic field suggests that multiple regions are simultaneously active in response to the presentation of language-related material. Therefore, additional assumptions would be required to solve the inverse problem, for instance to constrain source modeling with a limited number of fixed dipoles. This does not seem useful for the present data set as relatively large interindividual variability in the neuroanatomical location of language areas (Ojemann, 1983) would require additional measurements to define reasonable constraints. Furthermore, there is currently no procedure available that allows for localization of sites of neural oscillations without assumptions about a phase reference or alignment (Feige, 1999). Such assumptions would limit the validity of the approach. To examine the tentative conclusions in detail, future research using a whole head system and other combinations of tasks within one experiment is necessary.

In conclusion, the comparison of evoked and induced brain magnetic activity around $200 \mathrm{~ms}$ after stimulus onset showed that the two measures may reflect different elements of cognitive processing during a given task. The functional role of the languagerelated $60-\mathrm{Hz}$ effect that appears around $200 \mathrm{~ms}$ after stimulus onset and that has been observed in an earlier study by Eulitz et al. (1996) was specified. The general conclusion of the earlier study that this enhancement of the normalized spectral power seems to reflect oscillatory pattern specific to the processing of words can be maintained tentatively for sensors over the frontal-temporal scalp regions. When using measures from a sensor array that covers the major portion of the surface over the left hemisphere, the power of event-related oscillations in the $60-\mathrm{Hz}$ band was correlated with the familiarity of the visual Gestalt.

\section{REFERENCES}

Allison, T., McCarthy, G., Nobre, A., Puce, A., \& Belger, A. (1994). Human extrastriate visual cortex and the perception of faces, words, numbers, and colors. Cerebral Cortex, 5, 544-554.

Elbert, T. (1998). Neuromagnetism. In W. Andrä \& H. Nowak (Eds.), Magnetism in medicine (pp. 190-262). New York: Wiley.

Eulitz, C., Elbert, T., Bartenstein, P., Weiller, C., Müller, S., \& Pantev, C. (1994). Comparison of magnetic and metabolic brain activity during a verb generation task. NeuroReport, 6, 97-100.

Eulitz, C., Eulitz, H., \& Elbert, T. (1997). Differential outcomes from magneto- and electroencephalography for the analysis of human $\operatorname{cog}$ nition. Neuroscience Letters, 227, 185-188.

Eulitz, C., Maess, B., Pantev, C., Friederici, A., Feige, B., \& Elbert, T. (1996). Oscillatory neuromagnetic activity induced by language and non-language stimuli. Cognitive Brain Research, 4, 121-132.

Feige, B. (1999). Oscillatory brain activity and its analysis on the basis of $M E G$ and EEG. Münster, Germany: Waxmann.

Fiez, J. A., \& Petersen, S. E. (1998). Neuroimaging studies of word reading. Proceedings of the National Academy of Sciences of the United States of America, 95, 914-921.

King, J. W., \& Kutas, M. (1998). Neural plasticity in the dynamics of human visual word recognition. Neuroscience Letters, 244, 61-64.

Kuriki, S., Takeuchi, F., \& Hirata, Y. (1998). Neural processing of words in the extrastriate visual cortex. Cognitive Brain Research, 6, 193-203.

Kutas, M. (1997). Views on how the electrical activity that the brain generates reflects the functions of different language structures. Psychophysiology, 34, 383-398.

Kutas, M., \& Van Petten, C. (1988). Event-related brain potential studies of language. In P. K. Acles, J. R. Jennings, \& M. G. H. Coles (Eds.), Advances in psychophysiology (Vol. 3, pp. 139-187). Greenwich, CT: JAI Press.

Makeig, S. (1993). Auditory event-related dynamics of the EEG spectrum and effects of exposure to tones. Electroencephalography and Clinical Neurophysiology, 86, 283-293.

Nobre, A. C., Allison, T., \& McCarthy, G. (1994). Word recognition in the human inferior temporal lobe. Nature, 372, 260-263.

Ojemann, G. A. (1983). Brain organisation for language from the perspective of electrical stimulation mapping. Behavioral and Brain Sciences, $26,189-230$

Oldfield, R. C. (1971). The assessment and analysis of handedness: The Edinburgh inventory. Neuropsychologia, 9, 97-113.

Petersen, S. E., Fox, P. T., Snyder, A. Z., \& Raichle, M. E. (1990). Activation of extrastriate and frontal cortical areas by visual words and word-like stimuli. Science, 249, 1041-1044.
Posner, M. I., Sandson, J., Dhawan, M., \& Shulman, G. L. (1989). Is word recognition automatic? A cognitive-anatomical approach. Journal of Cognitive Neuroscience, 1, 50-60.

Price, C. J., Wise, R. J. S., \& Frackowiak, R. S. J. (1996). Demonstrating the implicit processing of visually presented words and pseudowords. $\mathrm{Ce}$ rebral Cortex, 6, 62-70.

Pulvermüller, F. (1996). Hebb's concept of cell assemblies and the psychophysiology of word processing. Psychophysiology, 33, 317-333.

Pulvermüller, F., Birbaumer, N., Lutzenberger, W., \& Mohr, B. (1997). High-frequency brain activity: Its possible role in attention, perception and language processing. Progress in Neurobiology, 52, 427-444.

Pulvermüller, F., Lutzenberger, W., \& Birbaumer, N. (1995). Electrocortical distinction of vocabulary types. Electroencephalography and Clinical Neurophysiology, 94, 357-370.

Reicher, G. M. (1969). Perceptual recognition as a function of meaningfulness of stimulus material. Journal of Experimental Psychology, 81, 275-280.

Salmelin, R., Service, E., Kiesilä, P., Uutela, K., \& Salonen, O. (1996). Impaired visual word processing in dyslexia revealed with magnetoencephalography. Annals of Neurology, 40, 157-162.

Schendan, H. E., Ganis, G., \& Kutas, M. (1998). Neurophysiological evidence for visual perceptual categorization of words and faces within $150 \mathrm{~ms}$. Psychophysiology, 35, 240-251.

Stippich, C., Freitag, P., Kassubek, J., Sörös, P., Kamada, K., Kober, H., Scheffler, K., Hopfengärtner, R., Bilecen, D., Radü, E.W., \& Vieth, J. B. (1998). Motor, somatosensory and auditory cortex localization by fMRI and MEG. NeuroReport, 9, 1953-1957.

Tallon-Baudry, C., \& Bertrand, O. (1999). Oscillatory gamma activity in humans and its role in object representation. Trends in Cognitive Sciences, 3, 151-162.

Van Petten, C., \& Senkfor, A. J. (1996). Memory for words and novel visual patterns: Repetition, recognition, and encoding effects in the eventrelated brain potential. Psychophysiology, 33, 491-506.

Wheeler, D. D. (1970). Processes in word recognition. Cognitive Psychology, 1, 59-80. 